\title{
Performance zootechnique des jeunes ovins mâles nourris en complémentation au résidu de moringa (Moringa oleifera Lam.) au Niger
}

\author{
Mahamadou ABDOU MALAM ${ }^{1 *}$, Abdou GOMMA DAN ${ }^{1}$, Salissou ISSA ${ }^{1}$, \\ Gambo YAHOUSSA ${ }^{1}$, Moctar KARIMOU ${ }^{2}$, Salifou BAGNAN ${ }^{2}$ et \\ Yaou Zakari MOUSSA ${ }^{2}$
}

\author{
${ }^{1}$ Institut National de Recherches Agronomiques du Niger (INRAN) BP 429 Niamey, Niger. \\ ${ }^{2}$ Mercy Corps - Maradi, Niger. \\ *Auteur correspondant ; E-mail : mamalam1@yahoo.fr; Tél: (+227) 968886 75, (+227) 90011322
}

\begin{tabular}{lll}
\hline Received: 07-06-2021 & Accepted: 10-09-2021 & Published: 30-10-2021 \\
\hline
\end{tabular}

\section{RESUME}

La présente étude entre dans le cadre de l'amélioration de la production animale en particulier les petits ruminants à travers l'augmentation de la disponibilité et la qualité de l'alimentation animale. Elle a pour but d'évaluer trois régimes alimentaires issus d'une combinaison de résidus de moringa (Moringa oleifera Lam.), des tiges de sorgho et le son du blé. Les ingrédients sont séchés et broyés séparément avant la formulation des aliments composés. Durant 90 jours, dix-huit jeunes ovins répartis en trois lots ont subi le test. Chaque lot correspondant à un traitement recevait un régime alimentaire : la ration alimentaire journalière est de $900 \mathrm{~g} /$ animal servie en deux repas et l'abreuvement s'est fait également deux fois par jour. Les échantillons d'ingrédients et d'aliments ont été prélevés et analysés, ainsi que les paramètres de croissance ont été collectées et analysées. Les résultats obtenus montrent que les résidus séchés de moringa ont la même teneur en MAT que la fane d'arachide ou les feuilles de niébé. Aucune différence significative n'a été prouvée pour tous les paramètres de performance de croissance étudiés entre les traitements. En l'occurrence le gain de poids vif total variait de 1,88 à 2,35 $\mathrm{kg}$ et le gain du poids moyen quotidien (GMQ) de 20,83 à 26,11 g tandis que l'indice de consommation (IC) se situait entre 2,9 à 3,7 kg de MS par kg de croît. Les producteurs de cette zone peuvent, à l'avenir, collecter, sécher, stocker les résidus de moringa et les utiliser comme fourrage à l'instar des résidus de récolte de niébé ou d'arachide.

(C) 2021 International Formulae Group. All rights reserved.

Mots clés : Résidus de Moringa, tige de sorgho, ovins, performance de croissance, Niger.

\section{Zootechnical performance of young male sheep fed as a supplement to moringa residue (Moringa oleifera Lam.) in Niger}

\begin{abstract}
The present study falls within the framework of improving animal production, in particular small ruminants, through increasing the availability and quality of animal feed. It aims to evaluate three diets derived from a combination of residues of moringa (Moringa oleifera Lam.), Sorghum stalks and wheat bran. The ingredients were dried and crushed separately before the formulation of compound feed. During 90 days, eighteen
\end{abstract}


young sheep divided into three groups underwent the test. Each batch corresponding to a treatment received a diet: the daily food ration is $900 \mathrm{~g} /$ animal served in two meals and the watering was also done twice a day. Samples of ingredients and food were taken and analyzed, as well as growth parameters were collected and analyzed. The results obtained show that the dried moringa residues have the same MAT content as peanut haulm or cowpea leaves. No significant difference was proven for all growth performance parameters studied between treatments. In this case the gain in total live weight varied from 1.88 to $2.35 \mathrm{~kg}$ and the gain in mean daily weight (ADG) from 20.83 to $26.11 \mathrm{~g}$ while the consumption index (CI) is was between 2,9 to 3,7 $\mathrm{kg}$ DM per $\mathrm{kg}$ growth. Producers in this area can, in the future, collect, dry, store moringa residues and use them as fodder like cowpea or peanut crop residues.

(c) 2021 International Formulae Group. All rights reserved.

Keywords: Moringa residues, sorghum stalk, lamb, growth performance, Niger Republic.

\section{INTRODUCTION}

Moringa oleifera Lam., appelé simplement moringa est un arbuste pouvant atteindre 5 à $10 \mathrm{~m}$ de hauteur (Asante et al., 2014). Originaire de l'Inde, au sud de la chaîne de 1'Himalaya (Foidl et al., 2001; Pandey et al., 2011), le moringa a été introduit dans toutes les régions tropicales et subtropicales et s'est vite adapté aux conditions climatiques de plusieurs pays africains (Fuglie, 2002).

Moringa oleifera Lam., est une plante à usage multiple (Popoola et al., 2013 ; Asante et al., 2014 ; Rabo et al., 2015). En Asie ce sont les fruits qui forment la partie la plus importante de M. oleifera, alors qu'en Afrique, l'on préfère les feuilles. Popoola et al. (2013) ont inventorié différents usages de moringa au Nigeria: alimentaire, médicinal, fourrager, bois de chauffe, floculant, et haie vive. Au Niger $M$. oleifera est un excellent légume feuille. Les feuilles de moringa se consomment cuites comme des épinards ou préparées en soupes ou en salade (Saint Sauveur et Hartout, 2001 ; Ratnadass et al., 2011). Elles sont fortement consommées par la majorité des ménages nigériens lors des rencontres cérémoniales, durant le mois de Ramadan, dans les restaurations lors des ateliers de travail. $M$. Oleifera est aussi utilisé dans la pharmacopée traditionnelle (Zerbo et al., 2007 ; Nguemo Dongock et al., 2018). Les feuilles de moringa font partie des légumes à fort potentiel nutritionnel. Elles sont très riches en vitamines $\mathrm{A}$ et $\mathrm{C}$, en $\mathrm{Ca}$, en Fer et en protéines, mais contiennent également tous les Acides Aminés essentiels (Foidl et al., 2001; Fuglie et al., 2002 ; Manzo et al., 2016 ; Koroney et al., 2018).

Au Niger, la production des feuilles de moringa est estimée à plus 90000 tonnes par an avec un rendement d'environ 18,7 tonnes /ha (INS, 2015). La région de Maradi est l'un des bassins de production de moringa surtout dans la commune de Djirataoua avec une production annuelle estimée à près de 12000 tonnes de feuilles fraîches/an (INS, 2015). Au cours de la récolte, les feuilles et les tigelles sont arrachées et après avoir séparé les feuilles pour la consommation humaine, il reste une importante quantité de tigelles qui constituent des résidus et peuvent être récupérées pour servir de compléments alimentaires pour les animaux, en particulier les petits ruminants.

Le Niger, étant un pays où le déficit en fourrage est récurrent (Abdou, 2017), l'alimentation du bétail est une problématique majeure pour l'élevage. Le présent travail entre dans le cadre de l'augmentation du disponible alimentaire pour bétail à travers la valorisation des résidus de moringa par son incorporation dans la ration des animaux. Ainsi, il s'est agi d'évaluer l'effet de ce résidu de moringa sur les performances zootechniques des jeunes ovins mâles.

\section{MATERIEL ET METHODES}

\section{Matériel}

\section{Site expérimental}

L'étude a été conduite dans le village de Djirataoua, chef-lieu de commune portant le même nom. Cette localité est située entre $7^{\circ} 14^{\prime} \mathrm{E}$ de longitude et $13^{\circ} 41^{\prime} \mathrm{N}$ de latitude, le 
long d'une vallée appelée « Goulbi Maradi » et se trouve dotée d'un périmètre irrigué d'une superficie de 650 ha. Cette infrastructure constitue un atout majeur dans le développement des activités agro-sylvopastorales de la localité avec une grande importance des cultures irriguées et l'élevage des ruminants domestiques.

\section{Aliments utilisés}

Les résidus de culture de moringa qui sont des brindilles ou tigelles défoliées ont servi de complément alimentaire aux ovins dont l'aliment de base est constitué des tiges de sorgho. En effet, la culture du moringa fait partie des cultures phares sur le périmètre irrigué de Djirataoua. Elle est source d'importantes quantités de résidus qui peuvent être valorisées dans l'alimentation des animaux d'élevage. Quant aux tiges de sorgho, elles proviennent du sorgho cultivé aussi bien sur le périmètre que sur les terres dunaires pendant la saison des pluies. Pour fabriquer les aliments, les résidus de cultures (tiges de sorgho et tigelles de moringa) sont séchés puis broyés séparément avant d'être mélangés selon les formules alimentaires (Tableau 1). Ainsi trois types d'aliments ont été formulés dont F1, F2 et F3 (tableau 1).

\section{Matériel biologique}

Dix-huit (18) jeunes ovins mâles dont le poids vif moyen au démarrage est de 23,19 \pm 2,52 kg ont été utilisés. Ces animaux étaient des tout-venants achetés sur le marché du village de Djirataoua. Pour le traitement sanitaire, l'ensemble des animaux a été déparasité avec Ivermectine et vaccinés contre la pasteurellose et la peste des petits ruminants au démarrage. Au cours de l'expérimentation, certains animaux ont fait la diarrhée en présentant des signes de maladie. Le vétérinaire privé leur a administré l'antibiotique (Oxytétracycline 20\%) et antidiarrhéiques (Diaroban).

\section{Matériel de terrain utilisé}

Il s'agit du matériel comme le broyeur, du petit matériel servant à la fabrication de l'aliment (pelle, bâche, arrosoir, bassines, gants, masques, bottes), des pesons pour la pesée des aliments et le poids vif des animaux, des mangeoires et abreuvoirs qui sont en plastique.

\section{Méthodes \\ Dispositif expérimental}

Les dix-huit ovins identifiés sont répartis au hasard en trois lots de six individus. Après une période d'adaptation de 14 jours, chaque lot a été nourri à l'aide d'une alimentation issue des formules alimentaires (Tableau 2). Les animaux sont attachés au piquet et nourris individuellement sous un même hangar construit en matériaux locaux pendant 90 jours de l'essai.

\section{Distribution des rations et abreuvement}

Au sein de chaque lot, les animaux ont reçu individuellement dans des mangeoires en plastique une ration alimentaire de $900 \mathrm{~g}$ servie en deux repas par jour (matin et soir). L'eau de boisson a été servie à volonté également dans des abreuvoirs en plastique.

\section{Collecte des données}

\section{Echantillonnage des aliments}

Les trois constituants des aliments (tiges de sorgho, résidus de moringa et son de blé) et les aliments préparés ont été échantillonnés et analysés au laboratoire de nutrition animale (LANA) de l'Institut National de Recherche Agronomique du Niger (INRAN). Cette analyse consiste à déterminer le taux de la matière sèche (MS) contenu dans les différents ingrédients par le séchage à l'étuve à $103^{\circ} \mathrm{C}$ pendant 6 heures. Le pourcentage de la matière minérale $(\mathrm{MM})$ a été déterminé par la calcination au four à $350^{\circ} \mathrm{C}$ pendant 3 heures, celui de la cellulose brute $(\mathrm{CB})$ par la méthode de Weende ; l'extrait éthéré (EE) par l'extraction au solvant organique (éther de pétrole) et la matière azotée totale (MAT) par la méthode de Kjeldahl.

Pesée des aliments et des animaux

Les quantités d'aliments distribuées aux animaux ont été pesées avant d'être servies et les refus ont été collectés par individu, puis pesés chaque jour le matin avant le service de 
la ration du jour. Le contrôle de l'évolution du poids des animaux s'est fait par pesée de deux jours successifs par décade. Les paramètres zootechniques suivants ont été collectés et calculés. Il s'agit de: poids initial, gain de poids quotidien, poids final, gain de poids total des animaux et indice de consommation.

\section{Analyse statistique}

Les données collectées sont saisies sur la feuille Excel puis ont été analysées à l'aide de GENSTAT selon le modèle Linear General Model et la comparaison des moyennes a été faite avec la Least Significatif Difference (LSD) au seuil de $\alpha=5 \%$.

Tableau 1 : Formulation des aliments.

\begin{tabular}{llc}
\hline Formules alimentaires & Constituants & Quantité de constituants(g) \\
\hline Formule 1 (F1) & Tiges de sorgho & 600 \\
\cline { 2 - 3 } & Résidus de moringa & 0 \\
\cline { 2 - 3 } & Son de blé & 300 \\
\hline Formule 2 (F2) & Tige de sorgho & 400 \\
\cline { 2 - 3 } & Résidus de moringa & 200 \\
\cline { 2 - 3 } & Son de blé & 300 \\
\hline Formule 3 (F3) & Tige de sorgho & 300 \\
\cline { 2 - 3 } & Résidus de moringa & 300 \\
\cline { 2 - 3 } & Son de blé & 300 \\
\hline
\end{tabular}

Tableau2 : Répartition des animaux nourris à base des aliments préparés à Djirataoua, région de Maradi au Niger.

\begin{tabular}{|c|c|c|c|}
\hline Traitements & Nombre d'animaux & Ingrédients & $\begin{array}{l}\text { Quantité des } \\
\text { ingrédients (g) }\end{array}$ \\
\hline \multirow{3}{*}{$\begin{array}{c}\text { Traitement } 1 \\
\text { (T1) }\end{array}$} & \multirow{3}{*}{6} & Tiges de sorgho broyées & 600 \\
\hline & & Résidus de moringa broyé & 0 \\
\hline & & Son de blé & 300 \\
\hline \multirow{3}{*}{$\begin{array}{c}\text { Traitement } 2 \\
\text { (T2) }\end{array}$} & \multirow{3}{*}{6} & Tiges de sorgho broyées & 400 \\
\hline & & Résidus de moringa broyés & 200 \\
\hline & & Son de blé & 300 \\
\hline \multirow{3}{*}{$\begin{array}{c}\text { Traitement } \mathrm{T3} \\
\text { (T3) }\end{array}$} & \multirow{3}{*}{6} & Tiges de sorgho broyées & 300 \\
\hline & & Résidus de moringa broyés & 300 \\
\hline & & Son de blé & 300 \\
\hline
\end{tabular}


RESULTATS

Composition chimique des ingrédients des aliments utilisés

La composition chimique des constituants (Tableau 3) montre que le pourcentage de la MS est similaire pour les trois éléments analysés. Par contre, le pourcentage en matière minérale des tiges de sorgho est le triple de celui des résidus de moringa et le quadruple de celui du son de blé. La teneur en cellulose brute $(\mathrm{CB})$ des résidus de moringa est de $37,3 \%$, elle est de $32,6 \%$ dans les tiges de sorgho. Quant au pourcentage en matière azotée totale (MAT), il est plus important pour le son du blé (18\%) que pour les deux autres ingrédients où il est de $8,2 \%$ pour les tiges de sorgho et $9,8 \%$ pour les résidus de moringa.

\section{Performances d'ingestion et zootechniques des ovins mâles complémentés avec le résidu demoringa}

L'incorporation de résidu de moringa n'a pas eu d'effet significatif sur l'ingestion de la matière sèche. Les moyennes des différents traitements ne sont pas significativement différentes $(p>0,05)$ (Tableau 4). La quantité moyenne d'ingestion la plus faible $(763 \mathrm{~g} / \mathrm{j})$ a été obtenue avec le traitement 2 et la plus élevée avec le traitement $3(768 \mathrm{~g} / \mathrm{j})$.

Les poids moyens initiaux des animaux ne sont pas significativement différents $(\mathrm{P}>$ $0,227)$ entre les trois lots correspondant aux trois traitements (T1, T2 et T3), Les poids vifs moyens finaux des animaux et le gain de poids total n'ont pas connu une différence significative $(\mathrm{P}>0,351)$ pour le $1^{\text {er }}$ paramètre et (P> 0,821) pour le second entre les trois traitements. Quant au gain moyen quotidien (GMQ) et l'indice de consommation (IC), aucune différence significative n'a été observée également entre les traitements ( $\mathrm{P}>0,708$ pour GMQ et $\mathrm{P}>0,534$ pour IC) (Tableau 4).

Tableau 3 : Composition chimiques des constituants entrant dans la formulation des aliments pour nourrir ovins à Djirataoua, région de Maradi au Niger.

\begin{tabular}{lccccc}
\hline Ingrédients & $\begin{array}{c}\text { Matière } \\
\text { sèche }(\%)\end{array}$ & $\begin{array}{c}\text { Matière } \\
\text { minérale }\end{array}$ & $\begin{array}{c}\text { Cellulose } \\
\text { brute }\end{array}$ & $\begin{array}{c}\text { Matière Azotée } \\
\text { Totale }\end{array}$ & $\begin{array}{c}\text { Extrait } \\
\text { éthéré }\end{array}$ \\
\cline { 3 - 6 } Son de blé & $96 \pm 0,01$ & $6,1 \pm 0,00$ & $11,6 \pm 0,15$ & $18,0 \pm 0,31$ & 3,77 \\
\hline Tige de sorgho & $96 \pm 0,06$ & $24,0 \pm 0,40$ & $32,6 \pm 0,23$ & $8,2 \pm 0,01$ & 1,51 \\
Résidus de moringa & $95 \pm 0,13$ & $8,7 \pm 0,98$ & $37,3 \pm 0,05$ & $9,8 \pm 0,15$ & 2,27 \\
\hline
\end{tabular}

Tableau 4 : Performances de croissance des antenais nourris avec des rations contenant différents niveaux d'inclusion des résidus de Moringa à Djirataoua dans la région de Maradi au Niger.

\begin{tabular}{lcccc}
\hline \multirow{2}{*}{ Variables } & \multicolumn{3}{c}{ Traitements } & Probabilité \\
\cline { 2 - 5 } & T1 & T2 & T3 & \\
\hline Matière sèche totale ingérée $(\mathbf{k g})$ & $69,0 \pm 0,524$ & $68,7 \pm 0,494$ & $69,1 \pm 0,605$ & 0,938 \\
\hline Matière sèche totale ingérée $(\mathbf{g} / \mathbf{j})$ & $766,3 \pm 5,83$ & $762,8 \pm 5,492$ & $768,0 \pm 6,723$ & 0,938 \\
\hline Poids initial $(\mathbf{k g})$ & $24,1 \pm 0,63$ & $23,6 \pm 0,59$ & $25,7 \pm 0,73$ & 0,227 \\
\hline Poids final $(\mathbf{k g})$ & $26,0 \pm 0,91$ & $25,8 \pm 0,83$ & $28,0 \pm 1,05$ & 0,351 \\
\hline Gain de poids total kg) & $1,88 \pm 0,52$ & $2,2 \pm 0,49$ & $2,4 \pm 0,60$ & 0,821 \\
\hline Gain Moyen Quotidien $\mathbf{( g / j )}$ & $20,8 \pm 5,82$ & $24,8 \pm 5,48$ & $26,1 \pm 5,72$ & 0,708 \\
\hline Indice de consommation $(\mathbf{k g}$ & $3,7 \pm 0,38$ & $3,1 \pm 0,21$ & $2,9 \pm 0,36$ & 0,534 \\
MS/kg gain $\mathbf{P V})$ & & & & \\
\hline
\end{tabular}




\section{DISCUSSION}

\section{Composition chimique des constituants}

Les résultats de l'analyse chimique montrent que les constituants utilisés ont un pourcentage en MS supérieur à $90 \%$. Les pourcentages en MM du son de blé et résidu de moringa sont inférieurs à $10 \%$, alors que celui des tiges de sorgho est très élevé (24\%). Cette teneur élevée en MM des tiges de sorgho peut être due à la contamination de celles-ci par le sable lié au mode de récolte consistant à couper les tiges et les laisser au sol pendant 10 à 14 jours avant leur collecte et stockage. A l'exception des résultats des tiges de sorgho pour la MM, ceux de MS et de MM obtenus pour les autres ingrédients sont comparables aux résultats des analyses de Abdou et al. (2017), Gomma et al. (2017), Akourki et al. (2019). De par l'analyse de la CB, les tiges de sorgho et le résidu de moringa sont des constituants cellulosiques contenant respectivement $32,6 \%$ et $37,3 \%$ de $\mathrm{CB}$ comme les tiges de mil avec 35,8\% de CB rapporté par Gomma et al. (2017) ou la paille de brousse qui peut atteindre $43 \%$ de CB, (Akourki et al., 2019). Ainsi, les tiges de sorgho et le résidu de moringa sont considérés comme aliments grossiers pour les antenais qui contiennent moins de $10 \%$ de MAT. Par contre, le son de blé avec $18 \%$ de MAT, aussi riche que la fane d'arachides en azote (Akourki et al. 2019) ou la fane de niébé avec 14,69\% (Gnanda et al., 2015), est considéré comme aliment source d'azote pour les jeunes ovins au cours de cette expérimentation.

\section{Performances d'ingestion de la matière sèche}

L'ajout de résidus de moringa n'a pas significativement changé l'ingestion de la matière sèche entre des différents traitements. Cela peut être expliqué par la forte teneur en cellulose des rations et la faible teneur en MAT des fourrages testés. Ces résultats sont conformes au principe que les rations riches en cellulose sont encombrantes avec une faible digestibilité. Les moyennes d'ingestion sont supérieures à celles obtenues par Dimon et al.
(2020) avec les rations contenant 5 et $10 \%$ de feuilles de moringa.

\section{Performance de croissance pondérale des jeunes ovins mâles}

Les gains de poids moyens quotidiens (GMQ) obtenus par les animaux sont compris entre 20,83 à 26,11 g; Ces résultats sont inférieurs à ceux de Moncho et al. (2016), qui ont obtenu des GMQ de 18,33g/j à 58g/j selon les traitements dans une étude portant sur la complémentation par des blocs multi nutritionnels (BMN) des ovins Djalonké au Bénin. Ils sont également inférieurs à ceux rapportés par Gomma et al. (2017) qui ont trouvé des valeurs allant de 59 à $118 \mathrm{~g} / \mathrm{j}$ sur l'évaluation des formules alimentaires des blocs multi nutritionnels d'embouche ovine et ceux de Dimon et al. (2020) avec des valeurs de 41,7 à $64,4 \mathrm{~g} / \mathrm{j}$ en fonction du traitement sur la complémentation aux feuilles de $M$. oleifera des moutons Djalonké. Concernant les gains totaux de poids vifs, les résultats obtenus au cours de cette étude sont de $1,88 \mathrm{~kg}$ pour le traitement T1, 2,23 kg pour le traitement $\mathrm{T} 2$ et $2,35 \mathrm{~kg}$ pour $\mathrm{T} 3$. Ces résultats sont comparables à ceux de Dimon et al. (2020) avec des valeurs variant de $3,53 \mathrm{~kg}$ à $4,83 \mathrm{~kg}$ pour une expérimentation qui a duré 60 jours. L'indice de consommation (IC) est compris entre 2,9 pour T3 à 3,7 pour $\mathrm{T} 1$; il est proche de celui de Dimon et al. (2020) qui ont rapporté un IC variant entre 1,61 à 2,37 kg de MS par kg de crôit sur des moutons de race Djalonké complémentés avec des feuilles de M. oleifera.

\section{Conclusion}

L'incorporation de résidus de moringa dans les rations des jeunes ovins n'a pas permis d'améliorer significativement les performances de consommation de matière sèche ni celles de croissance pondérale. Les résidus séchés de moringa dont la teneur en matière azoté totale (MAT) ou protéine brute est de l'ordre de 9,8\% de MS et celle en CB $(37,3 \%)$, peuvent être considérés comme des aliments grossiers très intéressants pour l'alimentation des ruminants. Ils ont une composition chimique proche de celle de 
résidus des légumineuses cultivées (fane d'arachide ou fane de niébé) et présentent l'avantage d'être produits en quantité durant toute l'année. Afin de mieux apprécier l'efficacité des résidus de moringa sur les performances des petits ruminants, d'autres tests avec des lots recevant uniquement des résidus de moringa et des lots avec des mélanges contenant de proportions variables de résidus de moringa doivent être conduits. Comparés aux fanes de niébé ou d'arachide, des études sur les coûts d'opportunité peuvent mieux présenter l'avantage économique que pourrait procurer l'utilisation des résidus de moringa. Les producteurs de Djirataoua en particulier et ceux des autres zones productrices du moringa au Niger peuvent, à l'avenir, collecter, sécher, stocker et utiliser ou vendre les résidus de moringa comme fourrage grossier à l'instar des résidus de récolte de niébé, d'arachide ou de gousses de Fedherbia albida..Ces résidus de moringa constituent des ingrédients pouvant contribuer dans la formulation des blocs multi nutritionnels dans les mêmes proportions que les fanes de niébé ou d'arachide dont les prix sont très élevés à certaines périodes de l'année.

\section{CONFLITS D'INTERETS}

Les auteurs de ce manuscrit déclarent n'avoir aucun conflit d'intérêt de quelque nature que ce soit ni entre les auteurs euxmêmes, ni entre les auteurs et des tierces personnes. En effet, MAM et ADG ont élaboré le protocole, analysé les données, rédigé et corrigé le manuscrit. YG et IS ont participé à l'élaboration du protocole, la supervision de l'essai et la correction du manuscrit, alors que $\mathrm{KM}, \mathrm{BS}$ et MZY ont contribué au suivi et à la collecte des données sur le terrain.

\section{REMERCIEMENTS}

Nous tenons à remercier, l'ensemble du personnel de l'INRAN, de MERCY-CORPSNiger et les producteurs qui ont contribué à la réalisation de cette étude. Nos remerciements vont également à l'endroit de l'USAID et Feed The Future pour avoir financé ce travail.

\section{REFERENCES}

Abdou MM. 2017. Production fourragère et intensification de l'intégration agriculture - élevage en zone agricole au Niger, Thèse de doctorat d'université ( $\mathrm{PhD})$, Université Cheikh Anta Diop, Dakar, Sénégal, p158

Abdou MM, Issa S, Gomma AD, Sawadogo GJ. 2017. Analyse technico-économique des Aliments densifies sur les performances de croissances des boucs roux de Maradi au Niger. International Journal of Biological and Chemical Sciences, 11(1): 280-292. DOI: http://dx.doi.org/10.4314/ijbcs.v11i1.22

Akourki A, Daka ARS, Sidikou DI, Chaïbou I. 2019. Effets du tourteau des graines de Balanites aegyptiaca sur la consommation alimentaire et les performances zootechniques des cabris roux de Maradi. International Journal of Biological and Chemical Sciences, 13(7): 3355-3363. DOI:

https://dx.doi.org/10.4314/ijbcs.v13i7.30

Asante WJ, Nasare IL, Tom-Dery D, OchireBoadu K, Kentil KB. 2014. Nutrient composition of Moringa oleifera leaves from two agro ecological zones in Ghana. African Journal of Plant Science, 8(1): 65-71. DOI: 10.5897/AJPS2012.0727

Dimon E, Toukourou Y, Seidou AA, Worogo HS, Soule AH, Traore IA. 2020. Performances zoo-économiques des ovins Djallonké complémentés avec la feuille de Moringa oleifera au Centre du Bénin. Afrique SCIENCE, 16(5): 189202. http://www.afriquescience.net

Foidl N, Makkar HPS, Becker K. 2001. Potentiel de Moringa oleifera en agriculture et dans l'industrie. In Potentiel de développement des produits de Moringa atelier du 29 octobre - 2 novembre 2001. De Saint Sauveur Armelle, Appora Viviane, Besse François (eds) Dar es-Salaam, Tanzanie, 175-232.

Fuglie Lowell J. 2002. Le Moringa : une arme dans la lutte contre la malnutrition. In : Potentiel de développement pour les produits de moringa : atelier international, 29 octobre - 2 novembre 2001, De Saint Sauveur Armelle, Appora Viviane, Besse François (eds), Dar es Salaam, Tanzanie, 
Montpellier : CIRAD, 1 Cd-Rom ISBN 287614-516-2 International Workshop on Development.

Gnanda IB, Nignan M, Ouedraogo S, Wereme N'diaye A, Traore O, Sinon B. 2015. Influence d'une co-construction de rationnement amélioré sur les performances d'embouche ovine paysanne dans la commune rurale de Korsimoro au Burkina Faso. International Journal of Biological and Chemical Sciences, 9(3): 1544-1556. DOI: http://dx.doi.org/10.4314/ijbcs.v9i3.35

Gomma $\mathrm{AD}$, M'bareck M, Ayssiwede S, Salissou I, Mahamadou MA, Siddo S, Chanono M. 2017. Evaluation technique et economique des formules alimentaires de blocs multi nutritionnels d'embouche ovine au Niger. Agronomie Africaine, 29(1) : 1-11.

Institut National de la Statistique. 2015. Etude pour l'estimation de la production et de la commercialisation des feuilles de moringa oleifera, République du Niger ; $50 \mathrm{p}$.

Koroney AS, Boubacar AS, Djibo H, Kasso IA. 2018. Moringa oleifra dans la résilience et la sécurité alimentaire des populations de la commune urbaine de Tibiri Gobir au Niger. Revue des Etudes Multidisciplinaires en Sciences Economiques et Sociales (REMSES). REMSES N ${ }^{\circ} 9$ Octobre-Novembre 2018.

Manzo ML, Halidou DM, Hallarou M, Illo A, Rabani A, Donnen P, Dramaix M. 2016. Composition de la poudre des feuilles sèches de Moringa oleifera dans trois régions du Niger. African Journal of Food, Agriculture, Nutrition and Development, 16(4): 11432-11442. DOI: 10.18697/ajfand.76.15115

Montcho M, Babatoundé S, Houndonougbo MF, Guédou A, Chrysostome AMC, Aboh BA, Mensah GA. 2016. Performances zoo-économiques en milieu réel des ovins Djallonke complémentés par les blocs multi nutritionnels (BMN) au Bénin. J. Rech. Sci. Univ. Lomé (Togo), Série B, 18(4): 922.
Nguemo Dongock D, Laohudumaye Bonyo A, Mapongmestem PM, Bayegone E. 2018. Etude ethnobotanique et phytochimique des plantes médicinales utilisées dans le traitement des maladies cardiovasculaires à Moundou (Tchad), International Journal of Biological and Chemical Sciences, 12(1): 203-216, DOI: https://dx.doi.org/10.4314/ijbcs.v12i1.16

PandeyA, Pradheep K, Gupta R, Nayar ER, Bhandari DC. 2011. 'Drumstick tree' (Moringa oleifera Lam.): a multipurpose potential species in India. Genetic Resources and Crop Evolution, 58(3): 453-460. DOI: 10.1007/s10722-0109629-6

Popoola JO, Obembe OO. 2013. Local knowledge, use pattern and geographical distribution of Moringa oleifera Lam.(Moringaceae) in Nigeria. Journal of Ethnopharmacology, 150(2): 682-691. DOI: 10.1016/j.jep.2013.09.043

Rabo Y, Lawali S, Drame AY, Mahamane A. 2015. Analyse structurelle des systèmes agroforestiers à base de Moringa oleifera Lam. dans les vallées du fleuve Niger et du Goulbi de Maradi (Niger). International Journal of Biological and Chemical Sciences, 9(6): 2555-2565. DOI: http://dx.doi.org/10.4314/ijbcs.v9i6.4

Ratnadass A, Zakari-Moussa O, Salha H, Minet J, Seyfoulay AA. 2011. Noordablitealis Walker, un ravageur majeur du Moringa au Niger (Lepidoptera, Crambidae). Bulletin de la Société Entomologique de France, 116(4) : 401-404.

Saint Sauveur A. DE, Hartout G. 2001. Moringa culture and economy in Niger. In The Miracle Tree-the Multiple Attributes of Moringa, Fuglie LJ (ed.). CTA: Wageningen, The Netherlands; 2944.

Zerbo P, Millogo-Rasolodimby J, NacoulmaOuedraogo OG, Van Damme P. 2007. Contribution à la connaissance des plantes médicinales utilisées dans les soins infantiles en pays San, au Burkina Faso. International Journal of Biological and Chemical Sciences, 1(3): 262 -274. 